The Water Research Association commenced with an income of $£ 11,000$. This rose to more than $£ 20,000$, but the research needs of the water industry are, with the increasing demands for water, so pressing that the water undertakings have decided to double their subscription to the Research Association and the Department of Scientific and Industrial Research has offered grant-in-aid up to $£ 27,000$. With an anticipated income of $£ 72,000$ the Association is moving its laboratories from Surrey to a site on the Thames in Buckinghamshire, where with an increased staff it can augment its present programme of work on the coagulation process of water purification, leak detection and plastic pipes, by the addition of filtration studies, dewatering of sludge, biological and chemical analytical methods and algal growth control. It will also pursue some of the hydrological interests of the water industry.

\section{Science Information News}

The National Science Foundation is issuing a new bi-monthly bulletin, Science Information News (1, No. 1; February-March 1959. Published bimonthly. Annual subscription, 1.75 dollars. Single copies, 25 cents. Washington, D.C. : National Science Foundation, 1959. Obtainable from the Superintendent of Documents, U.S. Government Printing Office), to provide a medium for reporting new and improved methods of disseminating scientific information and news of activities in this field. The first issue (February-March 1959) deals with the establishment by the National Science Foundation of its Science Information Service, the appointment of the Science Information Council and the establish ment of the Federal Advisory Committee on Scientific Information and of a Research Advisory Service on Information Processing. There are, however, notes on the International Conference on Information Processing to be held in Paris during June 13-23, on the expanded programme planned by the Office of Critical Tables of the National Academy of SciencesNational Research Council, the study by the Columbia University Bureau of Applied Social Research of the flow of scientific information among scientists, and the study by the Case Institute of Technology of scientific communication among chemists. It is hoped that in future issues the bulk of the bulletin will comprise material contributed by readers and outside the Foundation's own activities in this field.

\section{Royal College of Science and Technology of Glasgow}

THE latest annual report of the Royal College of Science and Technology, Glasgow, records no major building developments in 1957-58, but of a number of alterations the largest was the conversion of St. Paul's Chureh for the use of the School of Navigation. This building has yielded accommodation of a high standard and the rooms previously occupied by the school have been taken over by the Department of Natural Philosophy. Full-time students increased from 1,508 in $1956-57$ to 1,662 , part-time day students from 1,119 to 1,349 , while evening students decreased from 2,346 to 2,109. Besides a list of titles of theses presented by 21 members awarded higher degrees by the University of Glasgow, and of those approved for the awards of the postgraduate diploma of the College, the report includes a list of recent publications by members of the College. Further information on research work in progress is given in the Research Report for the Session 1957-58, in which abstracts of most of the publications listed are appended to the summaries of the work in progress in the various Departments.

\section{Jubilee of Queen's University, Belfast}

I $\mathrm{T}$ is just fifty years since, under the Irish Universities Act of 1908, The Queen's College, Belfast, founded in 1845 as part of a government plan for the extension of university education in Ireland, was dissolved and The Queen's University of Belfast was established. The retention of the title 'Queen's' for the new university was intended to show continuity with the foundation of 1845 and to proclaim adherence to the principle of non-sectarian education on which the old Queen's College (and those at Cork and Galway) had been established. This continuity is reflected in the short history of the College and the University, written by Prof. J. C. Beckett, which has been issued as a booklet (Queen's : a short History of Queen's College, Belfast, and the Queen's University of Belfast. Pp. $10.6 d$. Belfast : Queen's University, 1959), although the developments of the past fifty years occupy about half the account.

A new building, called the David Keir Building, to accommodate the Departments of Engineering, Chemistry, Zoology and Botany, was opened by the Duke of Edinburgh on May 21. The plans for this extension were described in Nature of February 19, 1955 , p. 323.

\section{Museum Treatment of Weakened Fabrics}

IN the issue of the Museums Journal of January 1959, Mr. F. R. Beecher describes a method of conserving fragile woven fabric by heat-sealing to prepared nylon net, allowing the intimate overall support provided by adhesion, while preserving the intrinsic textile quality of flexibility, of which some vestige may survive even in the worst example. The nylon net used is described technically as 15 denier 100 per cent bobbin with a count of $42 / 43$ per sq. in. It is sold as 'Nylusion tulle'. Textile fabric thus mounted-full details are to be found in the article quoted-can be examined on both sides. The method is also reversible as a touch of acetone will dissolve the film on the nylon and allow it to be lifted away.

\section{Resin-bonded Coatings of Polytetrafluoroethylene}

THIN films of polytetrafluoroethylene on metal and other surfaces are in use because of their properties of low friction, stability at high temperatures, resistance to chemical attack and weak adhesion. Methods of fixing the film on to the base material involve sintering at $400^{\circ}$ C., and for this reason films on rubber, plasties, wood and certain metals have not easily been obtained. A dispersion of polytetrafluoroethylene, under the trade name 'Emralon' (Acheson Colloid, Ltd.), has now become available and enables a coating of the polymer to be resin-bonded at low temperatures to a variety of surfaces. The advantage of this coating over the pure polytetrafluoroethylene film is its applicability to heat-sensitive surfaces, although, in addition, stronger adhesion and greater resistance to wear and corrosion are claimed. Of the two grades available, one uses a phenolic resin binder which can be applied by air spray and requires curing for one hour at $150^{\circ}$ C. ; the second contains a thermoplastio resin binder and needs no heat curing, so that it can be applied to surfaces where baking, even at $150^{\circ} \mathrm{C}$., is impracticable. 